\title{
A PROPOS DE LA TABLE RONDE «APOPTOSE, SPERMATOGENESE, SPERMATOZOIDES»
}

\author{
Jean-Pierre DADOUNE \\ Département de Morphologie et Développement, $\mathrm{CHU}$ Saint-Antoine, Paris
}

Sans préjuger de l'ordre dans lequel les données ont été présentées par les différents orateurs, quelques notions essentielles méritent d'être dégagées.

L'apoptose ou mort cellulaire programmée est un processus fondamental impliqué dans le développement embryonnaire normal et, chez l'adulte, dans l'homéostasie tissulaire qui résulte de l'équilibre entre la prolifération et la disparition des composants des différentes populations cellulaires. Le dérèglement du processus apoptotique peut conduire à un large éventail de pathologies en relation avec une apoptose massive (maladies neuro-dégénératives, SIDA) ou au contraire avec une inhibition de l'apoptose (néoplasies, maladies auto-immunes) [7].

L'apoptose est opposée à la nécrose, mort cellulaire causée le plus souvent par des agressions exogènes. Les cellules qui meurent par nécrose éclatent en libérant dans le milieu extra-cellulaire les médiateurs pro-inflammatoires qu'elles contiennent. La phagocytose des fragments cellulaires post-nécrotiques par les cellules avoisinantes activent la production de radicaux libres qui vont amplifier les lésions préexistantes.

L'apoptose est morphologiquement caractérisée par la condensation et la marginalisation de la chromatine provoquées par la fragmentation intra-nucléosomique de l'ADN, la formation de bourgeonnements au niveau de la membrane plasmique, la ballonisation des mitochondries et l'apparition de corps apoptotiques, terme ultime de la dégradation cellulaire [9].

Une famille de protéines, incluant TNF $\alpha$ (Tumor necrosis factor $\alpha$ ), FasL (CD95 ligand, APO-1), une lymphotoxine, CD40 ligand, CD30 ligand, CD27 ligand, 41-BB ligand et TRAIL (TNF-Related Apoptosis Inducing Ligand), intervient dans l'induction exta-cellulaire de l'apoptose [15].

Les protéines intracellulaires de la famille Bcl-2, comportant à la fois des membres pro-apoptotiques (Bax, Bak,
$\mathrm{Bad})$ et des membres anti-apoptotiques (Bcl-2, Bcl- $\mathrm{X}_{\mathrm{L}}$, $\mathrm{Bcl}-\mathrm{W}$ ), sont considérées comme des régulateurs importants de l'apoptose dans divers systèmes cellulaires $[1,8$, 26].

Deux voies majeures d'induction de l'apoptose, mettant en jeu des molécules adaptatrices et des molécules effectrices, ont été décrites dans les cellules de mammifères :

- Voie des récepteurs de mort cellulaire : la liaison de FasL sur son récepteur Fas (CD95) permet, via l'activation de FADD (Fas- Associated Death Domaincontaining protein), le recrutement de procaspases 8 qui sont ensuite transactivées par clivage. Les caspases 8 activent à leur tour les caspases effectrices 3,6 et 7 .

- Voie mitochondriale : en réponse à des dommages de l'ADN, la protéine p53 activée induit l'expression du facteur pro-apoptotique Bax qui, par compétition avec les membres anti-apoptotiques de la famille Bcl-2, provoque la sortie du cytochrome $\mathrm{C}$ de la mitochondrie ainsi que celle de l'AIF (Apoptosis Inducing Factor). Dans le cytosol, le cytochrome C s'associe aux procaspases 9 et à l'A paf-1 pour former l'apoptosome, complexe de caspases initiatrices qui activent alors, par clivage, les caspases effectrices [5].

De nombreux substrats des caspases ont été recensés parmi lesquels les composants du cytosquelette et du noyau, diverses protéases et DNases ainsi que des protéines de voie majeure de signalisation comme la PKC, Raf1 ou MEKK [16]

\section{Correspondance :}

Pr Jean Pierre Dadoune, Service d'Histologie, Biologie de la Reproduction et Cytogénétique, EA 1533 "Génétique de la Reproduction Humaine", Hôpital Tenon, 4 rue de la Chine, 75020 Paris. Email jean-pierre.dadoune@tnn.ap-hop-paris.fr 
Les calpaïnes, protéases à activation calcium-dépendante, sont également impliquées dans le processus apoptotique [27]. Elles ont un certain nombre de substrats en commun avec les caspases, notamment des protéines du cytosquelette et de l'enveloppe nucléaire, des membres de la famille Bcl-2 et les procaspases elles-mêmes [2].

La mort cellulaire programmée se manifeste conjointement à la prolifération et à la differenciation des cellules germinales au cours de la spermatogenèse normale. Elle joue le rôle d'un système de contrôle de qualité de la production des spermatozoïdes [27]. Les spermatogonies et les spermatides rondes apoptotiques présentent toutes les caractéristiques morphologiques et biochimiques de la mort cellulaire programmée. Ce n'est pas le cas des spermatocytes et des spermatides allongées, en dépit de leur marquage par la déoxynucléotidyltransférase et l'annexine V [6].

Une première vague apoptotique des cellules germinales survient lors du déclenchement de la spermatogenèse, à la puberté. Elle aurait pour effet d'assurer le maintien d'un rapport numérique optimal entre cellules germinales et cellules de Sertoli [20]. Chez l'adulte l'apoptose peut affecter jusqu'à 75\% des spermatogonies [14].

Les facteurs endocrines, paracrines et juxtacrines, en synergie avec les protéines intracellulaires, assurent l'homéostasie des trois familles de cellules germinales [19] :

\section{SIGNAUX ENDOCRINES}

Le rôle déterminant des gonadotrophines et des androgènes sur la survie des cellules germinales est bien établi [22]. La déprivation en $\mathrm{LH}$ aboutit à l'apoptose des spermatocytes pachytènes et des spermatides rondes, alors que la déprivation en FSH conduit à l'apoptose des spermatogonies et des spermatocytes pachytènes [21].

\section{SIGNAUX PARACRINES}

Plusieurs facteurs assurent la survie des cellules germinales primordiales, en particulier le LIF, le Steel Factor (SF) et la BMP (bone morphogenetic protein)-4. Chez l'adulte, les BMP-8A et -8B élaborées par les spermatides rondes favorisent la survie des spermatocytes. Le produit du gène homologue du gène « hedgehog » de la drosophile (Dhh) secrété par la cellule de Sertoli, exerce, via la cellule de Leydig, un effet anti-apoptotique indirect sur les spermatocytes.

\section{SIGNAUX DÉLIVRÉS PAR CONTACT DIRECT}

Le système $\mathrm{SF} / \mathrm{c}$-kit est non seulement nécessaire pour la migration des cellules germinales primordiales (SF est exprimé à la surface des précurseurs des cellules de Sertoli) mais aussi durant la première vague de la spermatogenèse. En effet, l'incidence de l'apoptose dans les spermatogonies est élevée, au moment de la puberté, chez les souris kit-haplodéficientes, avec pour conséquence une diminution de la spermatogenèse chez les animaux adultes [4].

Il a été montré que le système Fas/Fas-L intervient dans la régulation de l'apoptose des cellules germinales mâles chez le rat, la souris [11] et l'homme [18].

\section{ACTIVATION DES PROTÉINES INTRACELLULAIRES}

Les protéines de le famille $\mathrm{Bcl}-2$ sont reparties distinctement dans les trois types de cellules germinales, suggérant un rôle spécifique de ces protéines dans les processus de différenciation et de maturation [17]. La surexpression de Bcl-2 ou le knock-out de Bax chez les souris transgéniques entraîne une forte augmentation du nombre des spermatogonies, ayant pour effet d'altérer les stades ultérieurs le la spermatogenèse $[10,20]$.

D'autres protéines comme BRCA1 et BARD1, co-localisées dans les noyaux des cellules germinales mâles et éventuellement impliquées dans les processus de réparation, pourraient induire l'apoptose en cas de dommage cellulaire.

L'incidence de l'apoptose dans les spermatocytes et les spermatides est élevée chez les hommes inféconds [25]. Au cours des anomalies de la spermatogenèse, le taux des cellules apoptotiques est d'autant plus élevé que l'arrêt de la spermatogenèse est plus précoce [12]. L'utilisation de marqueurs spécifiques des cellules de Sertoli embryonnaires et prépubères (vimentine, $\mathrm{AMH}$ ) a montré que le blocage de la spermatogenèse comme 1'hypospermatogenèse sont associés à une maturation sertolienne imparfaite [24]. Cependant, alors qu'un nombre élevé de cellules germinales apoptotiques est observé chez les patients azoospermiques [13] ou chez les souris transgéniques présentant une surexpression sélective de Bcl-2 dans les cellules somatiques testiculaires [28], aucun signe de mort cellulaire programmée n'est détecté dans les cellules de Sertoli matures.

La présence d'apoptose dans les spermatozoïdes éjaculés reste encore discutée. La microscopie électronique et la méthode TUNEL ne sont pas suffisantes pour identifier avec certitude la mort cellulaire programmée. En effet la fragmentation de l'ADN dans les spermatozoïdes pourrait être en relation avec une anomalie du processus de compaction de la chromatine au cours de la spermiogenèse (références in [3]).

Pour conclure, trois remarques peuvent être formulées : 
- La caractérisation de l'apoptose nécessite la mise en œuvre de plusieurs techniques. On dispose de diverses méthodes immunocytochimiques pour évaluer l'expression des oncoprotéines Bcl-2, Bax, ainsi que de Fas et FasL dans le testicule. Dans le cas du spermatozoïde éjaculé il est recommandé d'utiliser, conjointement à la méthode TUNEL, l'analyse en cytométrie des cellules marquées par l'annexine $\mathrm{V}$ fluorescente et aussi de s'assurer de l'absence d'anomalies de condensation de la chromatine. La détection de l'activation des caspases par des inhibiteurs couplés à un fluorochrome a été pratiquée sur des cellules en culture [23]. Il serait intéressant d'appliquer cette technique à l'étude de cellules germinales en suspension.

- La discrimination entre une amplification de l'apoptose et un dysfonctionnement de la prolifération cellulaire, notamment dans l'hypospermatogenèse idiopathique, est réalisable grâce à l'immunolocalisation du PCNA (Proliferating Cell Nuclear Antigen) dans les noyaux des spermatogonies et des spermatocytes I, en complément de la mise en évidence de l'apoptose.

- La cellule de Sertoli est un véritable « check-point »de la spermatogenèse. De la sorte l'appréciation de l'apoptose dans la lignée germinale ne saurait être complète sans la recherche de critères attestant l'activité fonctionnelle des cellules de Sertoli.

\section{REFERENCES}

1. ADAMS J.M., CORY S. : The Bcl-2 protein family : arbiters of cell survival. Science. 1998, $281:$ 1322-1326.

2. CHUA B.T., GUO K., LI P. : Direct cleavage by the calciumactivated protease calpain can lead to inactivation of caspases. J. Biol. Chem., 2000, 275 : 5131-5135.

3. DADOUNE J.P. : Qualité du gamète testiculaire. Andrologie, $2001,11: 133-141$

4. GUERIF F., CADORET V., RAHAL-PEROLA V. et al. : Apoptosis, onset and maintenance of spermatogenesis : evidence for the involvement of Kit in Kit-haplodeficient mice. Biol. Reprod., 2002, $67: 70-79$.

5. HENGARTNER M.O. : The biochemistry of apoptosis. Nature, 2000, $407: 770-776$.

6. HENRIKSEN K., PARVINEN M. : Stage-specific apoptosis of male germ cells in the rat: mechanisms of cell death studied by supravital squash preparations. Tissue Cell.,1998, $30: 692-701$.

7. HETTS S.W. : To die or not to die : an overview of apoptosis and its role in disease. J. Am. Med. Ass., 1998, 279 : 300-307.

8. HSU S.Y., HSUEH A.J. : Tissue-specific Bcl-2 protein partners in apoptosis : An ovarian paradigm. Physiol. Rev., 2000, 80 : 593-614.
9. KERR J.F., WINTERFORD C.M., HARMON B.V. : Apoptosis. Its significance in cancer and cancer therapy. Cancer, 1994, 73 : 2013-2026.

10. KNUDSON C.M., TUNG K.S., TOURTELLOTTE W.G., BROWN G.A., KORSMEYER S.J. : Bax-deficient mice with lymphoid hyperplasia and male germ cell death. Science, 1995, 270 : 96-99.

11. LEE J., RICHBURG J.H., YOUNKIN S.C., BOEKELHEIDE $\mathrm{K}$. : The Fas system is a key regulator of germ cell apoptosis in the testis. Endocrinology, 1997, $138: 2081-2088$.

12. LIN W.W., LAMB D.J., WHEELER T.M., LIPSHULTZ L.I., KIM E.D. : In situ end-labeling of human testicular tissue demonstrates increased apoptosis in conditions of abnormal spermatogenesis. Fertil. Steril., 1997, $68: 1065-1069$.

13. MARTINCIC D.S., KLUN I.V., ZORN B., VRTOVEC H.M. : Germ cell apoptosis in the human testis. Pflugers Arch., 2001, 442 : R159-160.

14. MILLIGAN C.E., SCHWARTZ L.M. : Programmed cell death during animal development. Br. Med. Bull., 1997, 53 : 570-590.

15. NAGATA S. : Apoptosis by death factor. Cell, 1997, $88: 355$ 365.

16. NICHOLSON D.W. : Caspase structure, proteolytic substrates, and function during apoptotic cell death. Cell. Death Differ., $1999,6: 1028-1042$.

17. OLDEREID N.B., ANGELIS P.D., WIGER R., CLAUSEN O.P.: Expression of $\mathrm{Bcl}-2$ family proteins and spontaneous apoptosis in normal human testis. Mol. Hum. Reprod., 2001, 7 : 403-408.

18. PENTIKAINEN V., ERKKILA K., DUNKEL L. : Fas regulates germ cell apoptosis in the human testis in vitro. Am. J. Physiol., 1999, 276 : E310-316.

19. PRINT C.G., LOVELAND K.L. : Germ cell suicide : new insights into apoptosis during spermatogenesis. Bioessays, 2000, $22: 423-430$.

20. RODRIGUEZ I,, ODY C., ARAKI K., GARCIA I., VASSALLI P. : An early and massive wave of germinal cell apoptosis is required for the development of functional spermatogenesis. Embo. J., 1997, 16 : 2262-2270.

21. SHETTY J., MARATHE G.K., DIGHE R.R. : Specific immunoneutralization of FSH leads to apoptotic cell death of the pachytene spermatocytes and spermatogonial cells in the rat. Endocrinology, 1996, $137: 2179-2182$.

22. SINHA HIKIM A.P., SWERDLOFF R.S. : Hormonal and genetic control of germ cell apoptosis in the testis. Rev. Reprod., $1999,4: 38-47$.

23. SMOLEWSKI P., BEDNER E., DU L. et al. : Detection of caspases activation by fluorochrome-labeled inhibitors : Multiparameter analysis by laser scanning cytometry. Cytometry, 2001, $44: 73-82$.

24. STEGER K., REY R., KLIESCH S., LOUIS F., SCHLEICHER G., BERGMANN M. : Immunohistochemical detection of immature Sertoli cell markers in testicular tissue of infertile adult men : a preliminary study. Int. .J. Androl., 1996, 19 : 122 128. 
25. TESARIK J., GRECO E., COHEN-BACRIE P., MENDOZA C.: Germ cell apoptosis in men with complete and incomplete spermiogenesis failure. Mol. Hum. Reprod., 1998, 4 : 757-762.

26. VAUX D.L., KORSMEYER S.J. : Cell death in development. Cell, 1999, $96: 245-254$.

27. WANG K.K. : Calpain and caspase : can you tell the difference? Trends Neurosci., 2000, $23: 59$.

28. YAMAMOTO C.M., HIKIM A.P., LUE Y. et al. : Impairment of spermatogenesis in transgenic mice with selective overexpression of Bcl-2 in the somatic cells of the testis. J. Androl., 2001, 22 : $981-991$. 